\title{
Threshold Phenomena in a Throbbing Complex Plasma
}

\author{
Maxime Mikikian, ${ }^{*}$ Lénaïc Couëdel, ${ }^{\dagger}$ Marjorie Cavarroc ${ }^{\ddagger}$ Yves Tessier, and Laïfa Boufendi \\ GREMI, Groupe de Recherches sur l'Energétique des Milieux Ionisés, UMR6606, \\ CNRS/Université d'Orléans, 14 rue d'Issoudun, BP6744, 45067 Orléans Cedex 2, France
}

(Dated: July 5, 2010)

\begin{abstract}
In complex plasmas, the trapped dust particle cloud is often characterized by a central dustfree region ("void"). The void induces a spatial inhomogeneity of the dust particle distribution and is at the origin of many intricate unstable phenomena. One of this behavior is the so-called heartbeat instability consisting of successive contractions and expansions of the void. This instability is characterized by a strong nonlinear dynamics which can reveal the occurrence of incomplete sequences corresponding to failed contractions. Experimental results based on high-speed imaging are presented for the first time and underline this threshold effect in both the dust cloud motion and the evolution of the plasma light emission.
\end{abstract}

PACS numbers: 52.27.Lw

Complex (or dusty) plasmas consist of solid bodies trapped in an ionized gas $[1,2]$. These media are easily produced in laboratory plasma discharges either by directly injecting calibrated grains or by forming dust particles using reactive gases [3] or material sputtering. A fine knowledge of this mixture of plasma and big (relatively to ions and electrons) dust particles is required as it is encountered in many fields such as astrophysics $[4,5]$, industry $[6,7]$ and fusion $[8,9]$.

In a plasma, dust particles acquire an electric charge that strongly determines the equilibrium between the various forces acting on them and globally on the dust cloud. The force balance usually leads to dust-free sheaths near the walls and electrodes. Interestingly, it can also give birth to a dust-free region near the plasma center. This void [10-21] is sustained by an equilibrium between an outward ion drag force and an inward electric force [11]. An accurate understanding of this region is of major interest as its existence is a serious issue for both theory and applications. It prevents any global homogeneous treatment of the dust cloud and of the plasma in theoretical models. Indeed, the system consists of a central dust-free plasma surrounded by a different plasma containing dust particles. From an experimental point of view, the void can lead to erroneous conclusions on the presence of dust particles as no signal can be detected by laser light scattering techniques [7]. It also raises the problem of the homogeneous and controlled deposition of dust particles for industrial applications [20].

In many experiments, the force balance sustaining the void is very unstable and can be broken by slight changes in the discharge conditions. The equilibrium break does not lead to the void disappearance but rather to selfexcited oscillations of the void size: the heartbeat instability [13, 16, 22-24] consisting of successive contractions and expansions of the void. Until now, no clear explanation of the underlying mechanisms exists. However, previous experiments showed that the contraction seems to be triggered by an enhancement of the plasma light emission in the void region [23]. It can be supposed that this enhanced emission corresponds to an enhanced ionization rate that favors the inward electrostatic force at the expense of the outward ion drag force.

Once developed, this instability can be turned off by changing the experimental conditions but it can also stop by itself or, to be more specific, by the slight evolution of still unidentified parameters not controlled by the experimenter. In this last case, before the complete stop is reached, a progressive extinction phase takes place. It is characterized by complex nonlinear transitions recently identified as mixed-mode oscillations (MMOs) [24]. As the instability evolves towards its end, it shows weakness signs characterized by the occurrence of more and more failed contractions. The term "failed contraction" means that the threshold for an usual contraction is not reached while the conditions seem favorable. Instead, a intermediate state takes place.

In this paper, we investigate the complex extinction phase of the heartbeat instability and in particular the occurrence of failed contractions. Optical analyses are performed for the first time thanks to a high speed camera. Failed contractions are evidenced on the dust cloud motion and on the temporal and spatial evolutions of the plasma light intensity.

These experiments are performed in the PKE-Nefedov reactor $[14,25]$ where a capacitively coupled radiofrequency $(13.56 \mathrm{MHz})$ discharge is created between two electrodes of $4 \mathrm{~cm}$ separated by $3 \mathrm{~cm}$. Argon is injected to a pressure around 1.6 mbar and a typical power around $2.8 \mathrm{~W}$ is applied. Dust particles are grown by sputtering a polymer layer deposited on the electrodes. We use a high-speed camera (Mikrotron MC1310) at 1789 frames per second to observe either the plasma glow (camera in front of the plasma) or the dust cloud. In this last case, we use a laser sheet to illuminate the dust cloud and an interference filter on the camera objective. The angle between the laser direction and the camera position is small $\left(\sim 20^{\circ}\right)$ in order to optimize collection of the scat- 


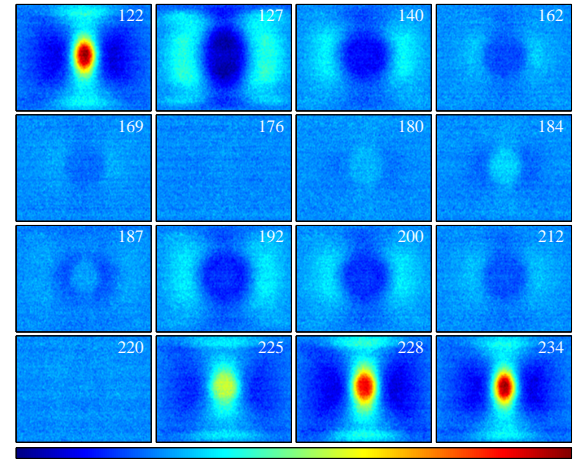

FIG. 1. (Color online) Evolution of the plasma luminosity during a contraction-expansion-contraction sequence containing 1 failed contraction (image 184). Color code is given in the colorbar at the bottom (false colors, from dark blue (low luminosity) to red (high luminosity) in arbitrary units). The image number is shown in the upper right corner. Image width and height correspond respectively to the electrode diameter and interelectrode distance. For each image, a reference frame (corresponding to an instant with a spatially homogeneous plasma emission) has been subtracted in order to improve contrast.

tered light. A few tens of seconds after plasma ignition, a visible three-dimensional dense cloud of grown dust particles (size of a few hundreds of nanometers) is formed [14]. This cloud usually exhibits a void region in its center. The heartbeat instability can start spontaneously or can be triggered by reducing the pressure and/or increasing the power. As this instability is self-excited and very sensitive, the conditions triggering the instability can vary from one experiment to another. Once started, the instability affects all plasma and discharge parameters and its signature can be followed on electrical and optical measurements [22-24]. High-speed imaging of the dust cloud motion during this phase is quite difficult to interpret due to the low image contrast and resolution, and especially to the complexity of the void expansion phase [23]. On the contrary, plasma light emission shows a clear and more obvious behavior. For the sake of clarity, we present first the plasma emission results and then those more intricate related to the dust cloud motion.

The void region is often characterized by a slightly higher brightness than the rest of the plasma [11, 18, 22, $26]$. The void contraction (motion of its boundary towards the center) corresponds to a strong enhancement of the plasma emission in the void region [23]. On the contrary, the void expansion corresponds to a dark center and brighter peripheral regions. This behavior is well observed in Fig. 1 showing the interelectrode plasma region during the instability. The main oscillation frequency (between real contractions) is about $16 \mathrm{~Hz}$. The presented series starts with a strong enhancement of the central plasma emission which is related to a real void contraction (image 122). This phase is followed by the void

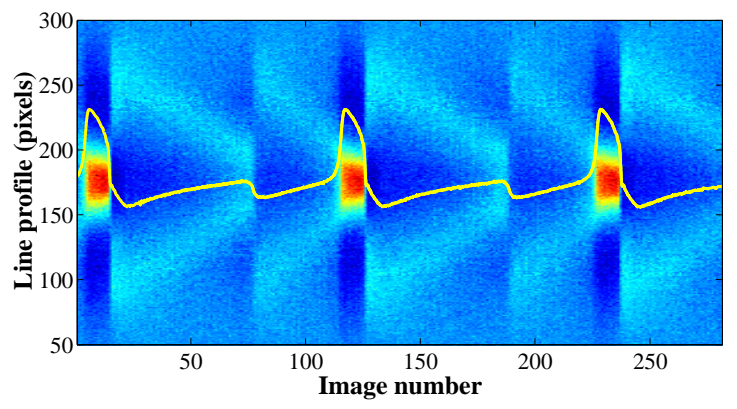

FIG. 2. (Color online) Spatiotemporal evolution of the instability shown in Fig. 1 (same image numbers). The y-axis corresponds to the electrode diameter. Electrical measurements are superimposed for comparison.

expansion where the central light emission is strongly reduced in comparison with the surrounding plasma. In particular, two symmetric bright regions are observed in the left and right parts (image 127). Up to image 184, the behavior is similar to a fully developed heartbeat instability previously studied in [23]: the plasma emission tends to homogenize and then starts to increase in the center. At this stage the central plasma emission is expected to accelerate its increase and to reach a value inducing the next contraction (as in image 122). On the contrary, the enhancement stops and the spatial distribution of the plasma emission follows the typical behavior of a new expansion (image 192) similar to image 127 but with less marked changes. The real contraction is then delayed and the strong enhancement of the plasma emission starts at image 225 . It reaches its maximum at image 234 where the real contraction occurs. Thus, it appears that failed contractions behave like usual ones, except that the emission enhancement is less marked. By using the classical notation of MMOs [24], the sequence in Fig. 1 corresponds to the $L^{S}$ state $1^{1}$ (1 main oscillation $L$ alternately with 1 small oscillation $S$ ).

This spatiotemporal behavior is easier to reveal by representing the whole plasma dynamics with one single image. This representative image is built by extracting the relevant information from each image. In this case, we assume that the main information is contained in the horizontal line passing through the void center [23]. By rotating all central lines and putting them side by side, we obtain the image depicted in Fig. 2. The image numbers on the $\mathrm{x}$-axis directly correspond to the numbers used in Fig. 1. A direct evidence of the occurrence of incomplete contractions is observed. These failed contractions clearly appear around image 75 and 184 (see also Fig. 1). After a real contraction (for example at $\mathrm{x}=122$ ), the regions of enhanced emission converge towards the center (between 130 and 184 with a speed of about $30 \mathrm{~cm} / \mathrm{s}$ ), as for a typical sequence, but once in the center (in 184) the enhancement is not able to increase more and thus to trigger a normal contraction. The sys- 


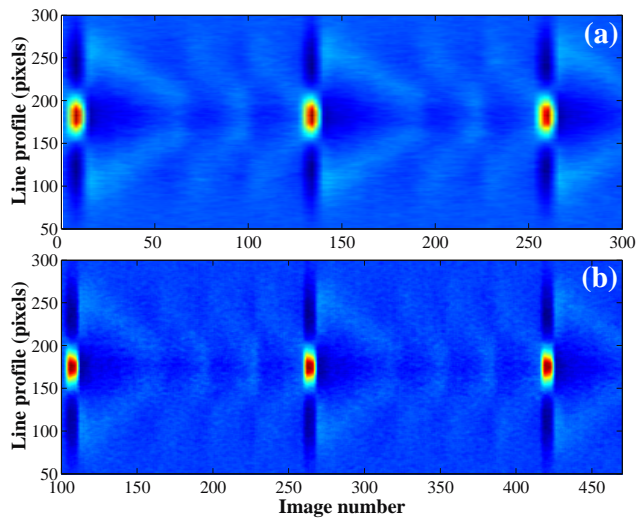

FIG. 3. (Color online) Similar analysis than in Fig. 2 for sequences containing (a) two and (b) three failed contractions.

tem goes back to a situation close to a normal void expansion and the convergence starts again (between 185 and 234). Then, between 225 and 234 the enhancement is really strong and the normal contraction occurs. It is also important to note that the duration of the convergence phase after the failed contraction is shorter than the one before. It means that the local conditions are different from those after a real contraction. It is confirmed by the observation that the regions of enhanced emission are less bright after the failed contraction. These failed events correspond to failed peaks in electrical or optical measurements [24]. It clearly appears by superimposing in Fig. 2 the amplitude of the discharge current.

As the instability is about to stop, more and more failed peaks (contractions) are observed [24]. By using the same type of analysis than in Fig. 2, we can observe this effect on the plasma emission (Fig. 3 obtained from another time series). These situations correspond to states $1^{2}$ and $1^{3}$, respectively in Figs. 3(a) and (b). The instability has a main frequency of about $14.3 \mathrm{~Hz}$ in Fig. 3(a) and of about $11 \mathrm{~Hz}$ in Fig. 3(b). The appearance of more failed peaks induces an increasing time in between two real contractions and thus tend to reduce the apparent main oscillation frequency [24]. Figure 3(b) also shows that, there is a very slight increase of the central luminosity for each new failed contraction. In the third failed contraction, the central glow enhancement is just below a given threshold and is stopped. When this threshold is exceeded, the central glow enhancement can increase quickly and strongly and then trigger a real contraction.

Failed contractions are harder to distinguish on the dust cloud. Indeed, in most cases, a new contraction occurs while the void re-opening is not completed and thus affects a cloud constituted of dust particles still in motion. As a failed contraction is weaker than a real one, the influence on the dust particle cloud is expected to be arduous to evidence without ambiguity. Figure 4 shows the dust cloud during a sequence with two failed

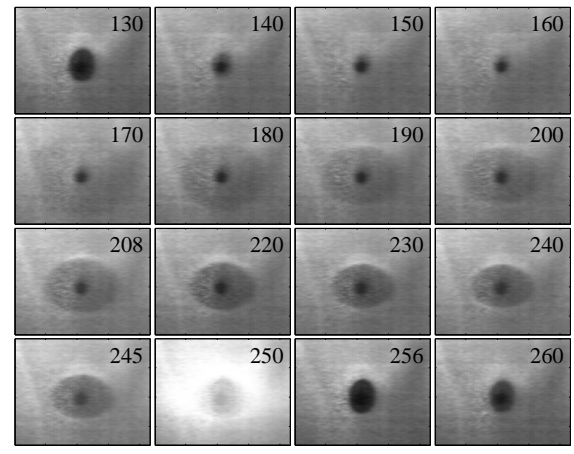

FIG. 4. Evolution of the dust cloud during a sequence with 2 failed contractions. Image number is indicated in each upper right corner. Failed contractions occur at images 170 and 208.

contractions (state $1^{2}$ ). The series begins during a real contraction (image 130) and the void minimum size is reached around image 150 . Then, the evolution of the completely empty central part of the void is little marked: main changes occur close to the bright plasma regions surrounding the void (as in image 127 of Fig. 1, see also [23]). These regions are not in the field of view of Fig. 4 which will provide new information when the symmetrical bright regions will converge towards plasma center (as shown in Figs. 1, 2 and 3). Dust particle motions are thus visible from image 170 . The two failed contractions occur at images 170 and 208 (better described later) and the real one at image 250. In this last case, the strong plasma light enhancement is also observed despite the presence of the interference filter on the camera.

To improve the analysis of Fig. 4, the same technique as in Fig. 2 is used to extract the global dust cloud dynamics (Fig. 5). In this case, the relevant information is considered to be contained in the central image column instead of the central line [23]. The correlation between Figs. 4 and 5 gives some insights on the dust cloud behavior during the two failed contractions. In Fig. 5, these

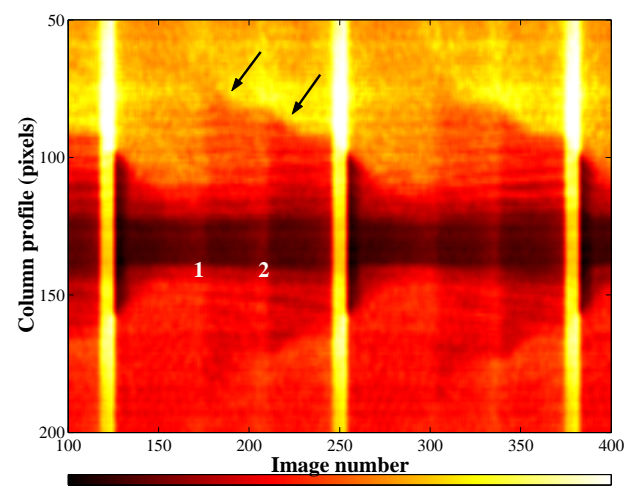

FIG. 5. (Color online) Global dust cloud motion obtained from the central columns of images of Fig. 4. Same image numbers are used. False colors, see colorbar at the bottom. 
failed events are marked with numbers 1 and 2 close to the central dark region representing the totally empty void. Two arrows mark detectable changes induced by these failed contractions. They seem to correspond to an accelerated motion towards the center of the identifiable interface delimiting two regions of different dust particle densities. These regions are better observed in Fig. 4 (for example in image 180) where three regions can be described: the totally empty void, a gray region with a low density of slow dust particles and a high density region. The interface discussed above delineates the last two regions. For each failed contraction this interface accelerates its motion towards the center as seen in Fig. 4 between 170 and 180, or 208 and 220. Figure 5 confirms that the main contractions occur when a strong enhancement of the plasma emission takes place inside the void. By looking at marks 1 and 2 it proves that failed contractions also correspond to a slight enhancement of the plasma emission in the void region but below a critical threshold and thus unable to trigger a real contraction.

For the fully developed heartbeat instability [23], the void contraction is triggered by a strong enhancement of the plasma emission in the void region. When this instability is just about to stop, failed events are observed on electrical and optical (by optical fibers) measurements and correspond to MMOs $[23,24]$. In this Letter, we evidenced for the first time these threshold phenomena on the evolution of both the global plasma emission and the dust cloud. On the plasma emission, failed events are almost similar to normal ones but are clearly characterized by variations with weaker amplitudes (Figs. 1, 2 and 3). The time required to reach a real contraction is shorter after a failed contraction than after a real one as shown in Figs. 2 and 3. It indicates that the system has been less perturbed and needs less time to reach the conditions for a real contraction. It can be also mentioned that the duration in between two failed contractions varies very little (Fig. 3). Concerning the dust motion, failed contractions appear as real contractions (motion towards the center), but they are weaker which is consistent with weaker changes in the plasma emission. Thus, an enhanced ionization rate (assumed to correspond to an enhanced plasma emission) in the void region corresponds to a decrease in the void size. It is the opposite situation to what is observed when the ionization rate is enhanced by, for example, increasing the power. This usually leads to an increase in the void size $[17,21,27]$. But, by increasing the power (through the electrodes) we certainly change the plasma in a global way. During the heartbeat instability the changes are self-induced and more localized in the center. This can lead to a different situation. Here, an enhanced ionization rate induces the void contraction by decreasing the ratio between the outward ion drag force and the inward electric force. It has been shown to be possible in the collisionless case in [13]. Nevertheless, in our case we are certainly in a collisional case as most of the time the void size is greater than the ionneutral mean free path. In [16] it has been shown that for a collisional case, an enhanced ionization rate can induce a decrease in the void size by taking into account the ion diffusion. Very recently, a fluid simulation taking into account the variations of the ionization [28] shows that above a certain threshold of the ionization rate, the void contracts. The results of the present paper will certainly support the approaches relating an enhanced ionization and a void contraction. It shows that it can only happen if a certain threshold for the ionization rate is reached. These new results can provide useful information on the physical mechanisms (especially the forces) involved in the void existence which is a still open challenging topic.

The PKE-Nefedov chamber has been made available by the MPE, Germany, under the funding of DLR/BMBF under grants No.50WM9852. ESA and Kayser-Threde $\mathrm{GmbH}$ are acknowledged for the high speed camera in the framework of the IMPACT program. This work was supported by CNES under contract 02/CNES/4800000059.

* maxime.mikikian@univ-orleans.fr

† Present address: Max-Planck-Institut für extraterrestrische Physik, Giessenbachstrasse, 85741 Garching, Germany

¥ Present address: Made In Dreux, 4 rue Albert Caquot, 28500 Vernouillet, France

[1] P. K. Shukla and B. Eliasson, Rev. Mod. Phys. 81, 25 (2009).

[2] G. E. Morfill and A. V. Ivlev, Rev. Mod. Phys. 81, 1353 (2009).

[3] J. Berndt et al., Contrib. Plasma Phys. 49, 107 (2009).

[4] C. K. Goertz, Rev. Geophys. 27, 271 (1989).

[5] G. Alcouffe et al., Plasma Sources Sci. Technol. 19, 015008 (2010).

[6] S. V. Vladimirov and K. Ostrikov, Phys. Rep. 393, 175 (2004).

[7] M. Mikikian et al., Eur. Phys. J. Appl. Phys. 49, 13106 (2010).

[8] S. I. Krasheninnikov et al., Plasma Phys. Control. Fusion 50, 124054 (2008).

[9] C. Arnas and A. A. Mouberi, J. Appl. Phys. 105, 063301 (2009).

[10] J. L. Dorier, C. Hollenstein, and A. Howling, J. Vac. Sci. Technol. A 13, 918 (1995).

[11] D. Samsonov and J. Goree, Phys. Rev. E 59, 1047 (1999).

[12] G. E. Morfill et al., Phys. Rev. Lett. 83, 1598 (1999).

[13] J. Goree et al., Phys. Rev. E 59, 7055 (1999).

[14] M. Mikikian et al., New J. Phys. 5, 19 (2003).

[15] K. Avinash, A. Bhattacharjee, and S. Hu, Phys. Rev. Lett. 90, 075001 (2003).

[16] S. V. Vladimirov, V. N. Tsytovich, and G. E. Morfill, Phys. Plasmas 12, 052117 (2005).

[17] A. M. Lipaev et al., Phys. Rev. Lett. 98, 265006 (2007).

[18] V. Land and W. J. Goedheer, New J. Phys. 9, 246 (2007).

[19] L. Couëdel et al., IEEE Trans. Plasma Sci. 36, 1014 (2008).

[20] M. Cavarroc et al., Phys. Rev. Lett. 100, 045001 (2008). 
[21] W. J. Goedheer, V. Land, and J. Venema, J. Phys. D: Appl. Phys. 42, 194015 (2009)

[22] M. Mikikian and L. Boufendi, Phys. Plasmas 11, 3733 (2004).

[23] M. Mikikian et al., New J. Phys. 9, 268 (2007)

[24] M. Mikikian et al., Phys. Rev. Lett. 100, 225005 (2008).

[25] A. P. Nefedov et al., New J. Phys. 5, 33 (2003).
[26] M. Mikikian et al., IEEE Trans. Plasma Sci. 36, 1012 (2008).

[27] V. N. Tsytovich, S. V. Vladimirov, and G. E. Morfill, JETP 102, 334 (2006)

[28] Z. Hu et al., Phys. Plasmas 16, 063707 (2009). 